\title{
Magnetic Domain Structure and Magnetization Reversal in Submicron-Scale Co Dots
}

\author{
A. Fernandez \\ M. R. Gibbons \\ M. A. Wall \\ C. J. Cerjan
}

This paper was preparcd for submittal to the 43rd Annual Conference on Magnetism and Magnetic Materials

Miami, Florida

November 9-12, 1998

September 24, 1998

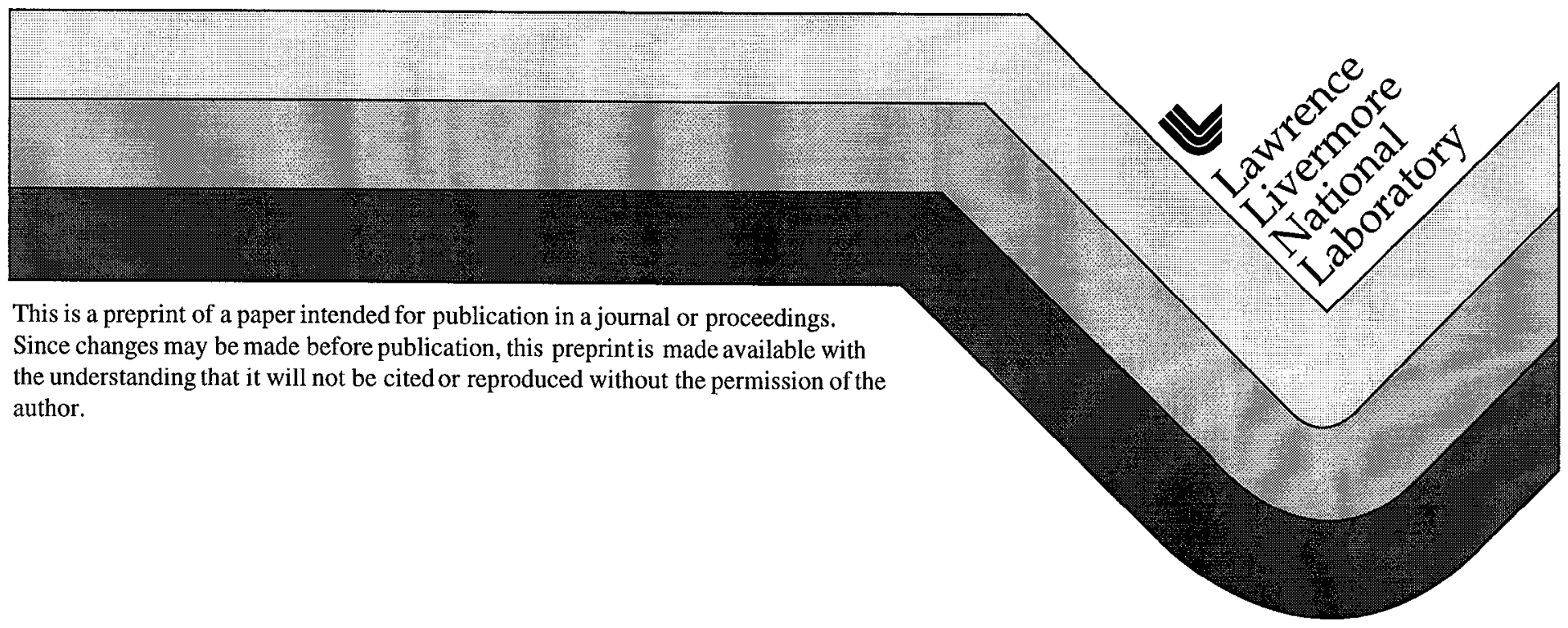




\section{DISCLAIMER}

This document was prepared as an account of work sponsored by an agency of the United States Government. Neither the United States Government nor the University of California nor any of their employees, makes any warranty, express or implied, or assumes any legal liability or responsibility for the accuracy, completeness, or usefulness of any information, apparatus, product, or process disclosed, or represents that its use would not infringe privately owned rights. Reference herein to any specific commercial product, process, or service by trade name, trademark, manufacturer, or otherwise, does not necessarily constitute or imply its endorsement, recommendation, or favoring by the United States Government or the University of California. The views and opinions of authors expressed herein do not necessarily state or reflect those of the United States Government or the University of California, and shall not be used for adverlising or product endorsement purposes. 


\title{
Magnetic domain structure and magnetization reversal in submicron-scale Co dots
}

\author{
A. Fernandez*, M.R. Gibbons, M.A. Wall, C.J. Cerjan \\ Luwrence Livermore National Laboratory, Livermore. CA 94551, USA
}

Received 14 May 1998; received in revised form 14 July 1998

\begin{abstract}
We present a magnetic force microscopy (MFM) analysis of atrays of submicron-scale Co dots fabricated by interference lithography. The dots are thin (180-300 $\dot{A})$ and elliptical in shape. MFM reveals that these structures relax into highly ordered remanent states whose symmetry and configuration are governed by their shape anisotropy. In particular, when the dots are saturated along their long-axis, a uniformly magnetized state persists at remanence. However, when the dots are saturated along their short-axis, they relax into a single-vortex state in which the circulation can have either sign. Both states are characterized by smoothly varying magnetization patterns and a high degree of uniformity across the array. We attribute the ordered behavior of these-structures to the film microstructure, which allows the shape anisotropy to dominate over magnetocrystalline anisotropy. By imaging a series of minor-loop remanent states, we show that magnetization reversal in these structures occurs yia the nucleation and annihilation of a single vortex. Magnetic hysteresis loop measurements are consistent with these observations and provide additional details. Furthemore, we present the results of micromagnetic simulations, which are in excellent agreement with both the MFM images and the hysieresis loop measurements. C) 1998 Elsevier Science B.V. All rights reserved.
\end{abstract}

\section{PACS:}

Keywords: Magnetic force microscopy; Co dots; Remanent states; Magnetization reversal; Micromagnetic simulations

\section{Introduction}

Recent interest in the magnetic properties of small, lithographically patterned magnetic structures has been motivated, in part, by the need to better understand the ultimate limits of magnetic

$$
510-720-320 a
$$

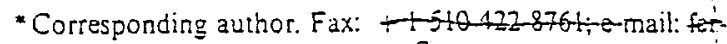

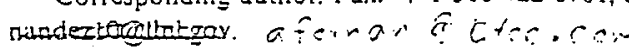
$\therefore$.

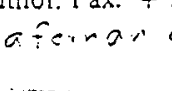

recording densities. In addition, the domain patterns and reversal mechanisms of small magnetic structures play an important role in the operation of magnetoresistive and giant-magnetoresistive sensors, paricularly as the size of these devices is pushed into the submicron regime where demagnetization effects are strong. The study of small magnetic structures also provides an excellent opportunity for testing micromagnetic simulations. These simulations, which can be used to model an 
arbitrary assembly of magnetic materials, are computationally intensive and therefore are easier to benchmark against structures that are small in size, relatively simple in shape, and have well-characterized composition.

The correlation of the magnetic properties of patterned structures to the size and shape of the structures is a central issue that has been addressed in many recent studies [1-6]. It is well known that, in the limit of large geometries, magnetic structures generally display complicated, multi-domain patterns. As the structure size is reduced, simpler domain patterns are formed [7]. Eventually, the single-domain state is reached when exchange energy dominates over all other energetic contributions. Reversal in single-domain particles is classically described by the Stoner-Wolihfarth (SW) model, which treats the reversal process as a coherent rotation of the magnetization [8]. For small $(\sim 50 \mathrm{~nm})$, low-aspect ratio dots, the SW model agrees well with the experimental data [9]. But the SW model is not generally applicable to all structures in which a uniformly magnetized state is formed at remanence. For example, bar-shaped magnetic particles have been shown to be singledomain below a critical bar width, but more complex reversal mechanisms are required to describe their hysteresis behavior $[2,10,11]$. Additional complications arise in structures where magnetocrystalline anisotropy plays a significant role [12].

In the following, we describe experiments on polycrystalline Co structures whose magnetic properties fall into an intermediate regime between single-domain and multi-domain behavior. The structures are thin (180-300 $\AA$ ), submicron in size, and are patterned with a uniaxial, in-plane, shape anisotropy. The saturation remanent states of the dots are analyzed by magnetic force microscopy (MFM). These measurements show that well-ordered remanent states are formed which are strongly dictated by the size and shape of the dots. We contrast our measurements with previously reported MFM studies of similar Co particles [13], and we relate the differences in the observed magnetic behavior to differences in the microstructure of the Co. In addition, we perform micromagnetic simulations to model the magnetic response of our dots and compare the results to the MFM images.
To gain insight into the reversal mechanism of the structures, we use MFM to image minor-loop remanent states. These measurements clearly demonstrate that reversal in these Co structures occurs by the nucleation and annihilation of a single vortex.

\section{Sample fabrication}

Arrays of magnetic dots are produced by interference lithography and a lift-off process [14]. Interference lithography is a robust patterning technique that allows the fabrication of arrays of sub-quarter micron size structures using optical illumination and standard resist processing. To achieve good lift-off profiles, a tri-layer process is used. Silicon wafers are coated with $200 \mathrm{~nm}$ of polymethylmethacrylate (PMMA), $20 \mathrm{~nm}$ of plasma deposited $\mathrm{SiO}_{2}$, an anti-reflective coating (ARC) and a layer of photoresist, in that order. The resist is exposed to an interference pattern formed at the intersection of two mutually coherent beams of $351.1 \mathrm{~nm}$ illumination. The period is controlled by adjusting the intersection angle and is fixed to $1 \mu \mathrm{m}$ for this study. After the first exposure, the sample is rotated $90^{\circ}$ in the exposure plane and exposed a second time. Development after this step would normally produce a two-dimensional array of resist dots [15]. Instead, the samples are treated to an image reversal step that produces an array of resist holes upon development. Details of the image reversal process are reported elsewhere [16]. The aspect-ratio of the holes is controlled by varying the relative doses of the two orthogonal exposures. After development, reactive ion etching is used to transfer the hole pattern through the $\mathrm{ARC}, \mathrm{SiO}_{2}$ and PMMA layers. The etch is followed by thermal evaporation of a thin layer of $\mathrm{Co}(180-300 \AA$ thick $)$. The Co is capped with $50-80 \AA$ of $\mathrm{Au}$ on some samples to prevent oxidation. Lift-off is performed in a solution of methylene chloride, which dissolves the PMMA layer.

A scanning electron micrograph of a fabricated array is shown in Fig. 1. The dots are elliptical in shape with an aspect ratio of 1.6. The structures have sharply defined edges and are highly symmetric about their long- and short-axes. We estimate 


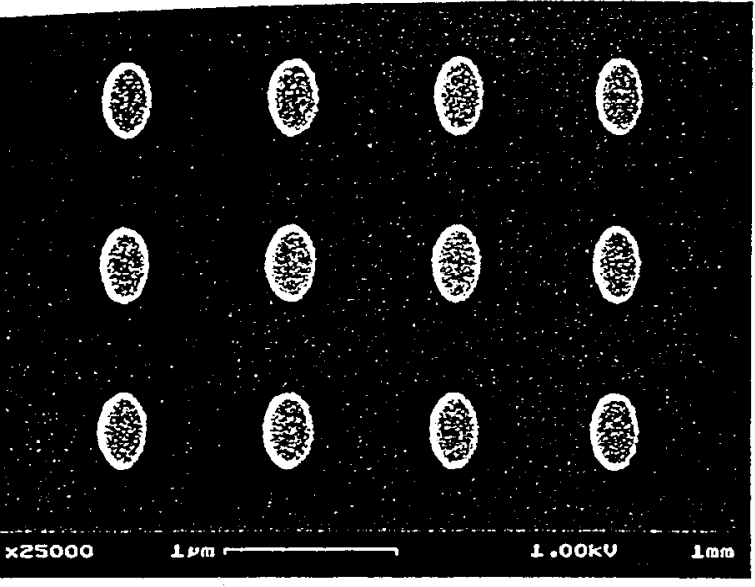

Fig. 1. Scanning electron micrograph of an array of polycrystalline Co dots patterned by interference lithography. The dots in this image measure $430 \mathrm{~nm} \times 270 \mathrm{~nm}$ and are centered on a $1.0 \mu \mathrm{m}$ grid.

the edge roughness to be less than $8 \mathrm{~nm}$. The size of the dots can be varied by adjusting the total exposure dose. In this paper, we present results on dots that are nominally $450 \mathrm{~nm} \times 250 \mathrm{~nm}$ in size. On a given sample, the dot size variation is less than $\pm 5 \%$ in a $1 \mathrm{~cm}^{2}$ area.

Transmission electron microscopy shows that the Co films consist of hcp grains with an average grain size of $60 \dot{\mathrm{A}}$. Diffraction measurements indicate that the $c$-axes of the grains are randomly oriented in all directions. The unpatterned films exhibit low coercivity values $(\sim 12 \mathrm{Oe})$, which can be directly attributed to the film microstructure. The effect of microstructure on the magnetic properties of the patterned structures will be addressed in detail below. Hysteresis loops of the dot arrays are measured using an alternating gradient magnetometer.

Magnetic force microscopy is carried out using a Nanoscope Dimension 5000. All the images presented are phase images acquired using the Lift Mode technique [17]. The MFM probe is magnetized perpendicular to the plane of the substrate. Prior to imaging the saturation remanent states of the arrays, the samples are placed in a uniform in-plane field of $4 \mathrm{kOe}$, which is subsequently ramped to zero. In Section 4, a more complicated magnetizing procedure is described.

\section{Saturation remanent states}

Magnetic force microscopy reveals that the Co dots relax into highly ordered remanent states whose symmetry is uniquely determined by their shape anisotropy. When $180 \AA$ thick dots are saturated along their long-axis, a uniformly magnetized state persists at remanence, as shown by the image in Fig. 2a. In this remanent state, the MFM images display a purely dipole character in which no other contrast is observed. Varying the tipsample separation does not change the observed pattern and only results in a change in the signal amplitude. The absence of fine scale structure in the images implies that the local magnetization $M$ of the dots is highly uniform on a length scale comparable to the MFM resolution $(\sim 20 \mathrm{~nm})$. The image in Fig. 2a also shows that the magnetization of the dots is closely aligned with their long-axes, which indicates that the easy-axis of magnetization is defined by the shape anisotropy. Furthermore, the large area image in Fig. $2 b$ shows that the response of the dots is remarkably uniform across the array. This result is important from a technological perspective. It demonstrates that a uniform remanent state can be generated in a large collection of patterned structures despite the inherent variability associated with the microfabrication process and with the strong magnetocrystalline anisotropy of the grains.

Although the easy-axis remanent state is a uniformly magnetized domain, the dots are not in a classical single-domain state, which would only be true if this configuration were the only stable magnetic state. When the dots in Fig. 2 are saturated along their short-axis, they relax into an entirely different remanent state, which has the distinctive MFM signature shown in Fig. 3. In this state, the images of the dots are divided into four equal quadrants that have alternating dark and light contrast. We interpret these images to indicate that a single magnetic vortex has formed at the center of each dot. Both signs of vortex circulation are represented in the array with equal probability. Later, we show more explicitly that the MFM image contrast is primarily derived from bulk magnetic charges, which are distributed within the dots in a quadrupole-like arrangement, and that surface 

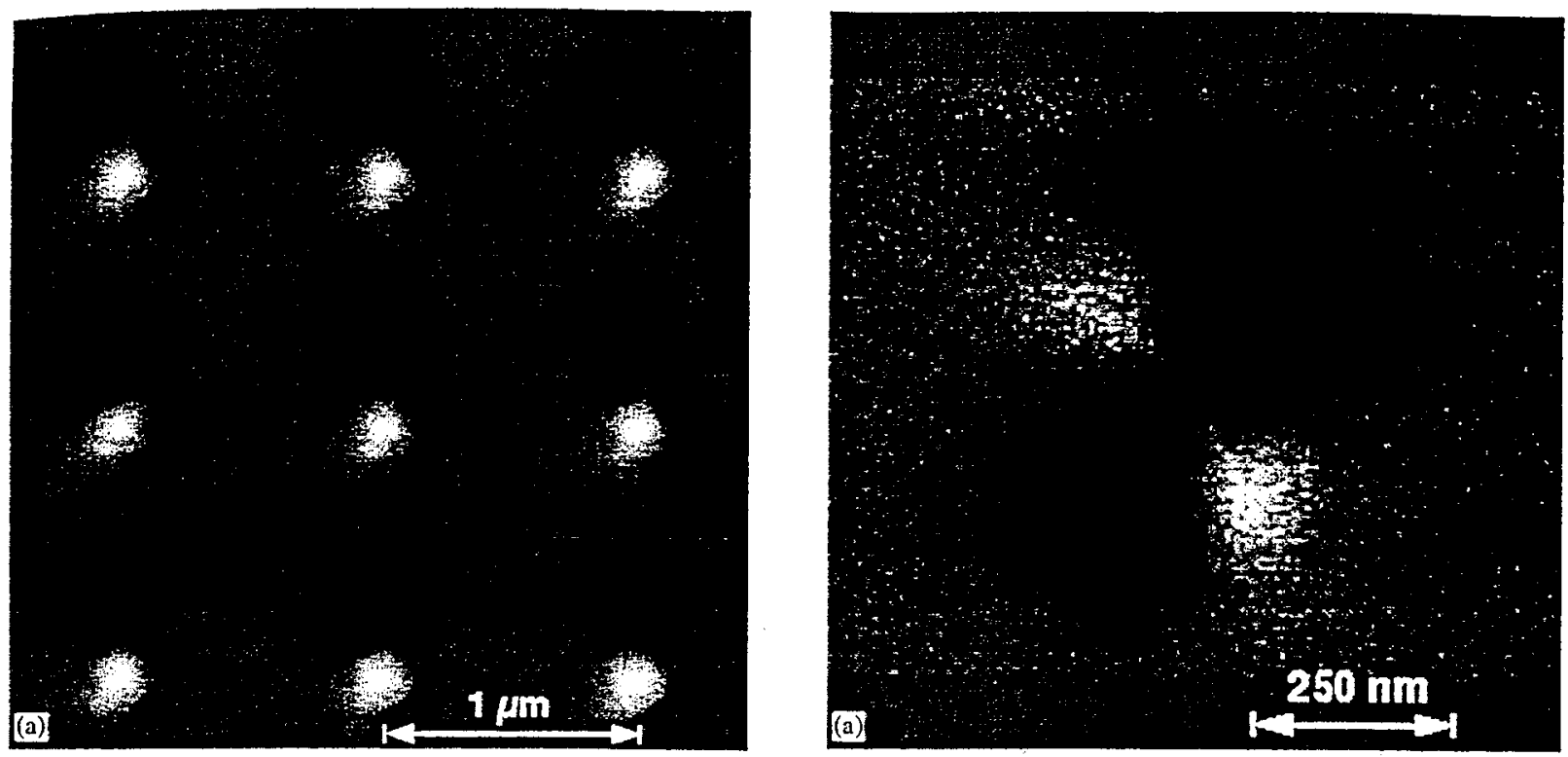

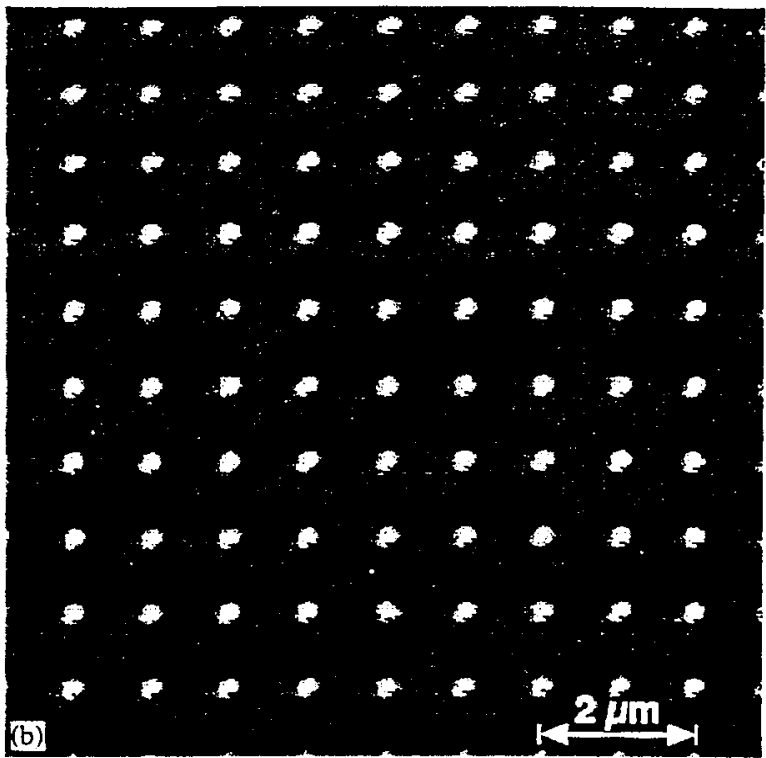

Fig. 2. (a) Magnetic force image of an array of $180 \dot{A}$ thick Co dots after saturating the dots along their long-axes and then removing the field. The image shows that the dots are uniformly magnetized parallel to the long-axis. (b) A larger scale image of the array in (a) illustrating the high degree of uniformity achieved across the array.

charges make a negligible contribution. Because the single-vortex state is centered about both axes, zero net magnetization is expected. This is confirmed by magnetic hysteresis measurements, which

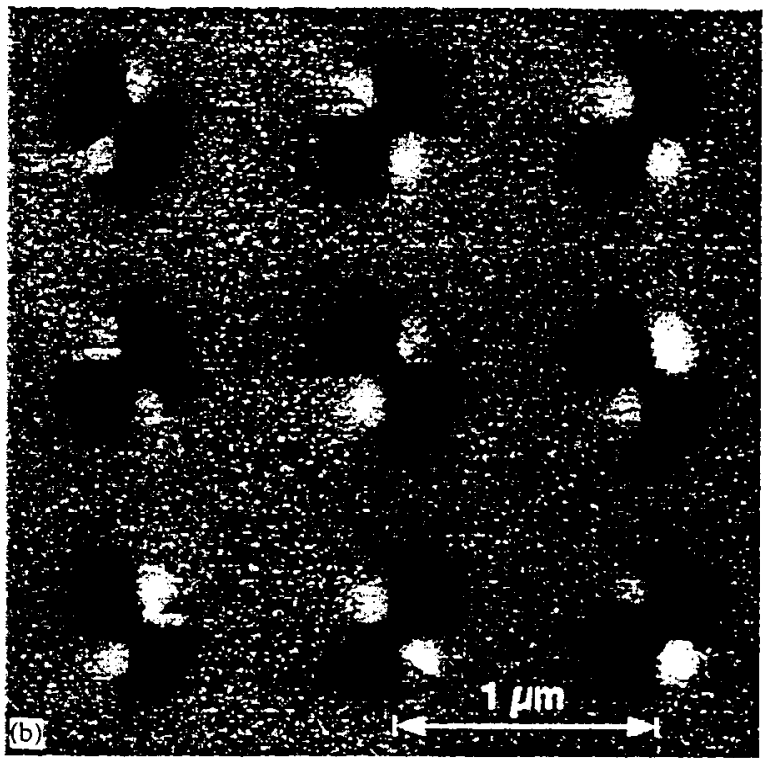

Fig. 3. (a) Magnetic force image of a single $180 \dot{A}$ thick Co dot after saturating the sample in the hard-axis direction and then removing the field. The contrast here indicates that the dot has relaxed into a configuration in which a single vortex occupies the center of the dot. (b) Magnetic force image of an array 180 $\dot{A}$ thick Co dots in the hard-axis remanent state.

are described in Section 5. The uniformity in response across the array is not as high as it is in the easy-axis state. We find that in this case $92 \%$ of the dots relax into the single-vortex pattern. The 
remaining $8 \%$ either remain in a uniformly magnetized state parallel to the easy-axis or they relax into a double-vortex structure.

It is important to point out that the gray scales in Figs. 2 and 3 have been adjusted to maximize the contrast in each image. On an absolute scale, the amplitude of the MFM signal is substantially weaker for the single-vortex state compared to the uniformly magnetized state (by a factor of $\sim 5$ ). The difference in signal simply reffects the fact that a much weaker stray field is produced by the nearly flux-closed, single-vortex configuration.

We have examined the behavior of thicker Co dots $(300 \AA)$ and observe that their saturation remanent states are very similar to the ones described above for the thinner dots except for two main differences. (1) In the easy-axis remanent state, the thicker dots cannot be readily imaged without the MFM tip disturbing the magnetic configuration. As the tip passes over these dots, they all initially appear similar to the dots in Fig. 2. But many dots irreversibly switch into a single-vortex state halfway through the scan. As hysteresis loops show (see Section 5), the onset of magnetization reversal is close to zero field in the $300 \AA$ Co dots. This makes the easy-axis remanent state relatively unstable in these dots, and therefore easily perturbed by the MFM probe. (2) In the hard-axis state, the response uniformity across the array is actually improved over the thinner dots. We find that in this case $97 \%$ of the dots relax into the single-vortex state. No dots are found in the uniformly magnetized state, and the other $3 \%$ support a double-vortex closure pattern. Both these differences can be qualitatively explained by the larger demagnetization field that exists in thicker Co dots, as will be discussed in more detail in Section 5.

To better interpret the MFM data, we simulated the magnetic response of the dots with a micromagnetic model [18]. The algorithm advances the magnetization using the Landau-Lifshitz-Gilbert dynamic equation in a semi-implicit formulation. The magnetostatic potential is solved using finite difference on a Cartesian, nonuniform mesh. Included in the simulation is the elliptical shape of the dots, exchange coupling $\left(A=1.6 \times 10^{-6} \mathrm{erg} / \mathrm{cm}^{2}\right)$, and uniaxial crystalline anisotropy $\left(K_{1}=1.0 \times 10^{6}\right.$ $\left.\mathrm{erg} / \mathrm{cm}^{3}\right)$. The crystalline axes are randomly oriented from grain to grain, and the mesh discretization is set equal to the average the grain size $(60 \AA)$. The evolution of the magnetization pattern over a full hysteresis loop is calculated using $50 \mathrm{Oe}$ steps. Simulated MFM images are generated by assuming the tip interacts with the sample as a fixed point-dipole, which is generally a good first approximation [19]. The image is rendered from a gray scale contour plot of the quantity $-\mathrm{d}^{2} H_{z} / \mathrm{d} z^{2}$, calculated approximately $100 \AA$ above the simulated dot.

While the size and grain structure of the simulated dots were based on measurement, the exchange and anisotropy constants were not known precisely. Bulk values were used and then varied slightly until a match with the measured hysteresis loop and MFM data was found. A simulated MFM image of the hard-axis remanent state is displayed in Fig. 4a. Comparison of the simulation to the data in Fig. 2 shows a close match. As can be seen in Fig. 4b, the simulation reveals that the four-quadrant image is characteristic of a single-vortex configuration with moments at the center forming a Néel-type core. Furthermore, the simulation shows that the direction of magnetization varies in a smooth and continuous fashion about the core. This behavior differs from the four sharply defined domain walls typically calculated [20] and observed [7] in rectangular particles. The simulation results, ported-in more-detail-elsewhere-[21], are also in good agreement with the easy-axis remanent state and in excellent quantitative agreement with the hysteresis loop measurements shown in Section 5.

In addition to describing the equilibrium domain configuration, the simulation results allow us to distinguish between surface and volume magnetic charge contributions to the MFM images. To a first approximation, the strength of the stray magnetic field above the dots is proportional to the total surface and volume charges per quadrant. The volume charge is calculated by integrating $\nabla \cdot M$ over one quadrant of the simulated dot, while the surface charge is computed from the surface integral of $\boldsymbol{M} \cdot \boldsymbol{n}$. We find that the total volume charge per quadrant exceeds the surface charge per quadrant by a factor of $10^{2}-10^{3}$, from which we conclude that the primary sourte of MFM image contrast is the
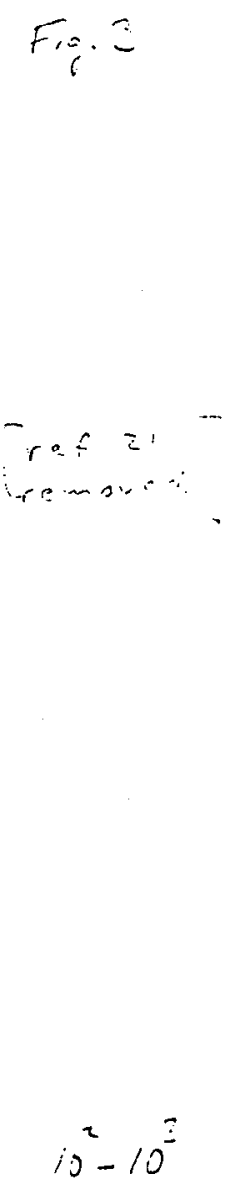

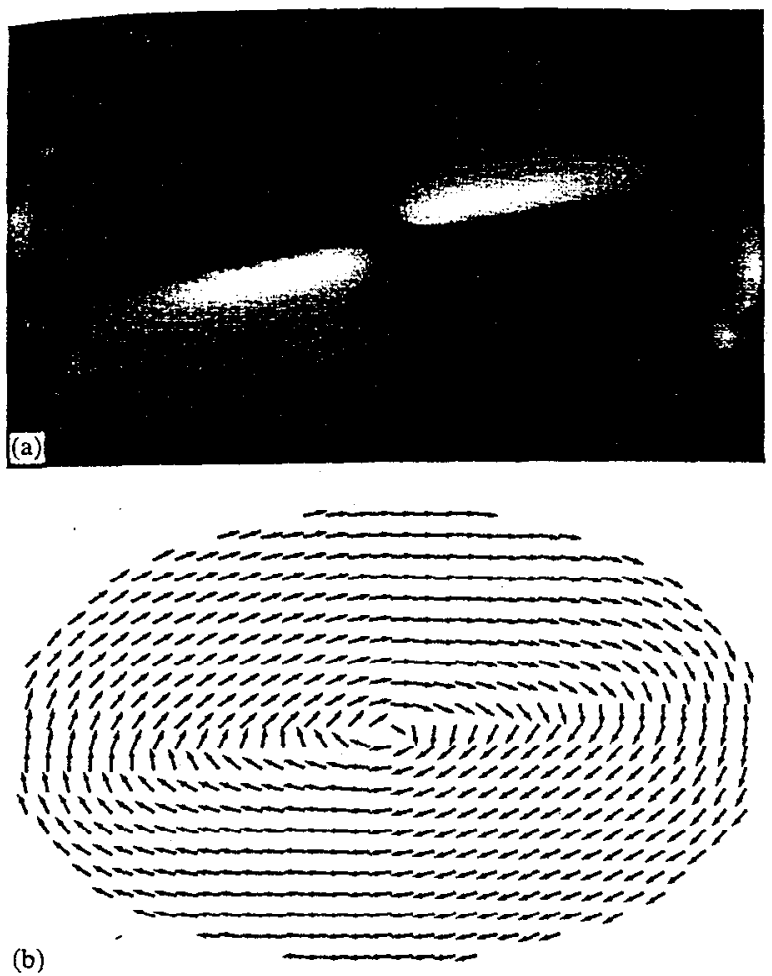

(b)

Fig. 4. Micromagnetic simulation results of the hardaxis remanent state. (a) A simulated MFM image of the hardaxis remanent state, which reproduces the same contrast seen in the experimental data. (b) The corresponding magnetization configuration showing a single-vortex domain pattern. The actual discretization used is two times finer than what is displayed.

volume charge. We note that this argument is based on the assumption that the fixed point-dipole approximation is indeed a valid description for the tip-sample interaction. Effects in which stray fields from the sample alter the MFM probe (or vice versa) are not taken into account.

The high degree of local uniformity exhibited by our Co structures is contrary to an earlier study by New et al. of similarly sized $(400 \mathrm{~nm} \times 200 \mathrm{~nm})$ Co particles fabricated by e-beam lithography [13]. Using MFM, they found that the easy-axis remanent state of their structures contained a great deal of local disorder, which they attributed to the strong magnetocrystalline anisotropy of the Co grains. Furthermore, hysteresis loop measurements in the hard-axis di- rection showed a large remanent magnetization. This is inconsistent with the formation of a nearly flux-closed, single-vortex state as we observe in our structures.

At first glance, the disparity in magnetic behavior between Co structures so closely matched in size and shape is surprising. However, the differences can be understood by considering the differences in the Co microstructure. In New's study, the Co was deposited by $\mathrm{DC}$ magnetron sputtering onto a $\mathrm{Cr}$ underlayer [13]. As a result, their films consisted of $100-200 \AA$ size HCP grains that were highly textured with the $c$-axis in-plane. By contrast, our films are made using thermal evaporation, which is a less energetic deposition process than sputtering. Consequently, our films have smaller grains $(\sim 60 \AA)$ and are not textured. The combined effect of smaller grains and lack of texturing size alters the relative balance between exchange and crystalline anisotropy energy. In the limit that grain sizes are large compared to typical domain wall widths, crystalline anisotropy will force the domains to align with the easy-axis of the crystallites, thereby causing local disorder in the direction of the magnetization. As grain sizes decrease, exchange energy rises in proportion to the domain wall density, which initially scales as the inverse square of the grain size. In the limit that grain sizes are small compared to domain wall widths, the intergrannular exchange coupling will dominate and will smooth out the effects of crystalline anisotropy. The domain wall width is approximately given by the expression $\pi \sqrt{A / K_{1}}$. This yields a typical wall width of $190 \AA$ in Co, which is comparable to the grain size in New's samples. The lack of in-plane texturing in our films further reduces the contribution of crystalline anisotropy to the energy. Thus we can expect our Co films to have comparatively stronger intergrannular exchange coupling. This is evident by the much lower coercivity of our films $(\sim 12 \mathrm{Oe})$ compared to the sputtered films in New's study $(\sim 350 \mathrm{Oe})$. The intergrannular coupling in New's films may be further weakened by diffusion of $\mathrm{Cr}$ to the grain boundaries from the $\mathrm{Cr}$ underlayer. While these arguments qualitatively explain the differences in the observed behavior, a more controlled experimental study would be valuable. 


\section{Magnetization reversal}

A common approach to study reversal processes using MFM is to measure a series of minorloop remanent states [22]. The structures are first saturated in an applied field. The field is then reduced to a fixed negative value $H_{\mathrm{m}}$ after which it is ramped to zero. The remanent state is then imaged in the MFM. This sequence is repeated multiple times, and each time the value of $H_{\mathrm{m}}$ is incremented. In cases where reversal occurs over a narrow field range, this procedure has been effective for determining the approximate switching field of the structures $[2,11]$. In addition, it may be possible to capture the structure in a stable, intermediate configuration, which can provide valuable clues to the reversal mechanism.

We have applied the minor-loop approach to determine the reversal mechanism in the $300 \AA$ thick Co dots in the easy-axis. We observe that for $H_{\mathrm{m}}$ in the range of $0 \mathrm{Oe}$ to $-200 \mathrm{Oe}$, the minorloop remanent states consist of a mixture of dots that are uniformly magnetized and dots that contain a single-vortex. The number of dots that have not nucleated a vortex decreases rapidly with increasing negative field. Below a field of $-300 \mathrm{Oe}$, nearly all of the dots have switched out of the uniformly magnetized state and into a single-vortex state (see Fig. 5). A small percentage of the dots, $\sim 3 \%$, nucleate a double-yortex closure pattern. (These dots are identified by an arrow in Fig. 5.) Increasing $H_{\mathrm{m}}$ to greater negative values has no effect on the remanent domain states until a second threshold is reached at $-900 \mathrm{Oe}$. At this point the single vortex pattern begins to disappear in some dots. Below a field of $-1000 \mathrm{Oe}$, all the dots have completely reversed to a uniformly magnetized state of ofpposite sign.

We can infer from the above observations that magnetic reversal in these structures occurs predominantly by the nucleation and annihilation of a single magnetic vortex. This well-ordered reversal process contrasts with the more varied and disordered reversal mechanisms observed for the sputtered Co structures discussed earlier [12]. The fact that in some ranges of field the minor-loop remanent states consist of a mixture of domain states

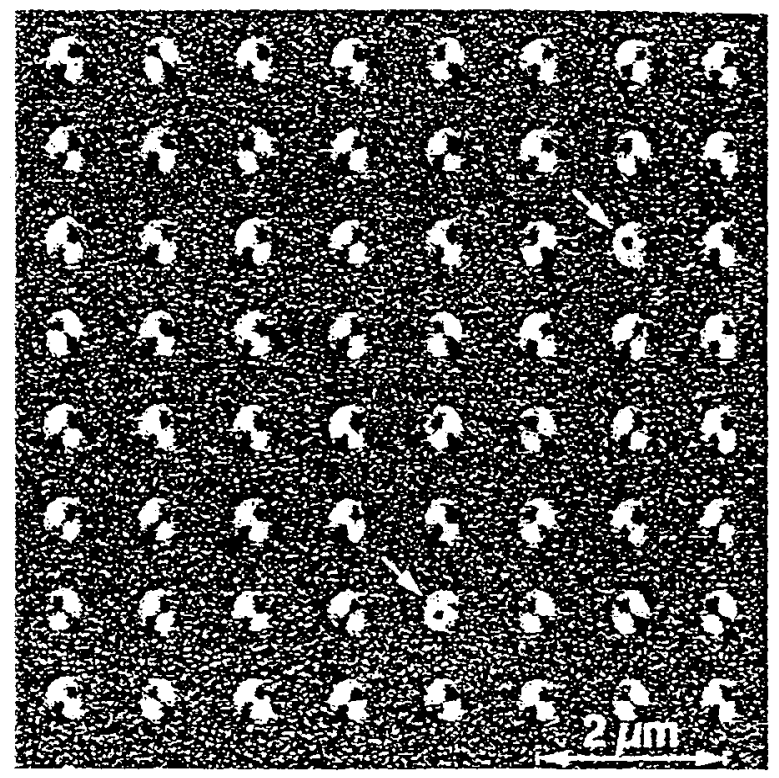

Fig. 5. Magnetic force image of an array of $300 \dot{A}$ thick dots in a minor-loop remanent state. The dots were first saturated in the easy-axis direction, and then a reverse field of -300 Oe was applied. This image provides strong evidence that the dots primarily reverse their magnetization in the easy-axis direction through a single-vortex nucleation and annihilation process. A small number of dots, identified by the arrows, reverse their magnetization via a double-vortex nucleation and annibilation process.

implies that critical fields for vortex nucleation and annihilation vary to some extent from dot to dot. Such variation may have several sources. Size variation, edge roughness, and the magnetocrystalline anisotropy of the grains may all contribute to a complicated energy surface that gives rise to a distribution of nucleation and annihilation fields. By sampling a large number of dots in a mixed minor-loop state, we may be able to correlate the magnetization pattern to variations in size as measured in the topographic part of the scan. Determining the relative contribution of edge roughness and crystalline anisotropy may only be possible through simulations. It is important to emphasize, however, that despite the variability in nucleation fields caused by different dot morphologies, nearly all of the dots in our arrays reverse their magnetization by the same exact mechanism. 


\section{Magnetic hysteresis loops}

Magnetic hysteresis loops of the patterned structures provide a complementary picture to the MFM analysis given above. In Figs 6 are the measured loops in the easy-axis direction and in the hard-axis direction, respectively, for an array of $300 \AA$ thick dots. The easy-axis loop shows a large remanence $\left(0.9 \mathrm{M}_{\mathrm{s}}\right)$ and a coercivity of $200 \mathrm{Oe}$. This represents an increase in coercivity of nearly 20 times over the sheet film coercivity. By contrast, the hard-axis loop shows zero remanent magnetization. Moreover, the hard-axis loop is closed over a central portion of the loop. These results are consistent with the formation of a nearly fluxclosed, single-vortex state at remanence. The closure of the loop near zero field implies that the vortices can move freely within the dots without being pinned by defects.

The hysteresis loops in Fig. 6 are very similar in shape to the ones measured by Wernsdorfer et al. for a single Co particle using a highly sensitive SQUID technique [23]. Their Co structures measured $300 \mathrm{~nm} \times 200 \mathrm{~nm}$ in size and were also produced by thermal evaporation. As their data and our data show, the easy-axis and hard-axis loops are characterized by two relatively abrupt changes in magnetization, which are marked by the symbols $H_{\mathrm{n}}$ and $H_{\mathrm{a}}$ in both graphs. From our MFM analysis, the significance of these transitions is clear. The first transition $H_{\mathrm{n}}$ marks the nucleation of a singlevortex at one edge of the dots, while the second transition $H_{\mathrm{a}}$ marks the annihilation of the vortex at the opposite edge. This interpretation was first suggested by Wernsdorfer et al. [23], and our MFM data confirms this simple picture. The only difference between our hysteresis loops and theirs is that the critical fields for nucleation and annihilation are not as sharply defined in our loops. The broader width of the transitions is further indication that a distribution of nucleation and annihilation fields exists in our dot arrays. In practice, we ascribe a particular value for $H_{\mathrm{n}}$ and $H_{\mathrm{a}}$ to a given array by finding the points of steepest decent in the transition regions.

From the hysteresis loops, one can see that the position of the nucleation (annihilation) field is shifted toward more positive (negative) values in
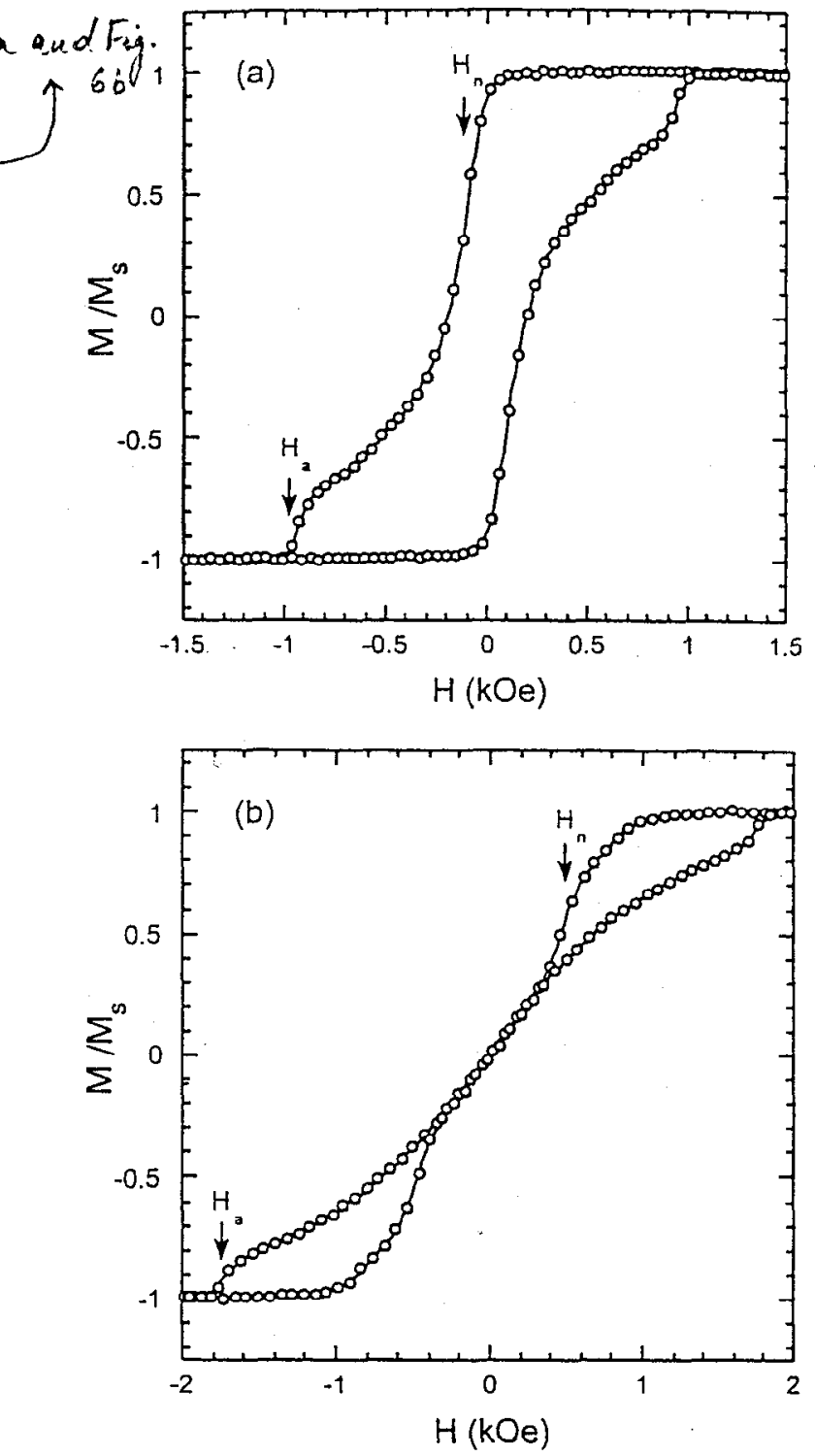

Fig. 6. Magnetic hysteresis loops for an array of $300 \AA$ thick Co dots measured with an alternating gradient magnetometer. The easy-axis loop in (a) shows a large remanence and a coercivity of $200 \mathrm{Oe}$. The hard-axis loop in (b) shows zero remanence, which is consistent with the formation of a single vortex. The transitions labeled $H_{n}$ and $H_{3}$ mark the fields at which nucleation and annihilation of the vortices occur, respectively.

going from the easy-axis to the hard-axis. We measure shifts of 540 and 770 Oe for the nucleation and annihilation fields, respectively, for $300 \AA$ thick dots. Wernsdorfer et al. attributed these shifts to 
the demagnetization field, which varies with the angle of the applied field [23]. While this explanation correctly estimates the magnitude of the shifts, it does not explain why they are different for the two types of processes. Nevertheless, the effect of the demagnetization field can also be seen by comparing the hysteresis loops for dots of varying sizes and thickness. Increasing the thickness, for example, results in a linear shift in the nucleation and annihilation fields, which can be directly correlated to the linear increase in the demagnetization field.

\section{Conclusions}

In summary, we have presented MFM measurements of arrays of submicron-scale Co dots fabricated by interference lithography. The data indicates that the magnetic response of these structures is dominated by the uniaxial shape anisotropy of the structures and not the magnetocrystalline anisotropy of the Co. This leads to the formation of highly ordered remanent states that are characterized by smoothly varying magnetization patterns and a high degree of uniformity across the array. The behavior exhibited by the dots contrasts with previously reported results on similarly sized Co structures, which showed greater local disorder in the remanent domain patterns. We attribute the higher degree of uniformity and symmetry in our structures to differences in the Co microstructure. Specifically, smaller grain sizes and the lack of texturing result in a stronger intergrannular exchange coupling, which mitigates the effects of crystalline anisotropy.

The MFM data are consistent with magnetic hysteresis measurements of the arrays, and both sets of data are in agreement with our micromag' netic simulations. Altogether, the MFM data, the hysteresis loops and the simulations provide a complete picture of the reversal mechanism in these structures. In this size regime, reversal in each dot is initiated by the nucleation of a single vortex at one end of the dot. As the applied field is reduced, the vortex is swept to the opposite end of the dot where, at a critical field, it is annihilated. The observed nucleation and annihilation fields are modulated by the demagnetization field, which depends on the angle of the applied field. The only significant distinction between reversal in the easyaxis and reversal the hard-axis is in the field values for nucleation and annihilation. The two different saturation remanent states (i.e. uniform magnetization and single-vortex) represent the two basic configurations that describe the reversal process.

\section{Acknowledgements}

The authors would like to thank Prof. Kenneth Verosub and the Department of Geology at the University of California, at Davis, for allowing us to use their alternating gradient magnetometer. We also thank Rodney Agayan for assisting with the interferometric lithography process development. This work was performed at the Lawrence Livermore National Laboratory for the Department of Energy under contract No. W-7405-Eng48.

\section{References}

[1] C. Miramond, C. Fermon, F. Rousseaux, D. Decanini, F. Carenac, J. Magn. Magn. Mater. 165 (1997) 500.

[2] R. O'Barr, S. Schultz, J. Appl. Phys. 81 (1997) 5458.

[3] M. Hehn, K. Ounadjela, J.P. Bucher, F. Rousseaux, D. Decanini, B. Bartenlian, C. Chappert, Science 272 (1996) 1782.

[4] L. Kong, S.Y. Chou, J. Appl. Phys. 80 (1996) 5205.

[5] R.M.H. New, R.F.W. Pease, R.L. White, J. Magn. Magn. Mater. 155 (1996) 140.

[6] A.B. Johnston, J.N. Chapman, B. Khamsehpour, C.D.W. Wilkinson, J. Phys. D 29 (1996) 1419.

[7] S. McVitie, J.N. Chapman, IEEE Trans. Magn. 24 (1988) 1778.

[8] E.C. Stoner, E.P. Wohlfarth, Philos. Trans. Soc. London A 240 (1948) 599.

[9] W. Wernsdorfer, K. Hasselbach, A. Benoit, G. Cernicchiaro, D. Mailly, B. Barbara, L. Thomas, J. Magn. Magn. Mater. 151 (1995) 38.

[10] W. Wernsdorfer, B. Doudin, D. Mailly, K. Hasselbach, A. Benoit, J. Meier, J.-P. Ansermet, B. Barbara, Phys. Rev. Lett. 77 (1996) 1873.

[11] M. Lederman, D.R. Fredkin, R. O'Barr, S. Schultz, M. Ozaki, J. Appl. Phys. 75 (1994) 6217.

[12] R.D. Gomez, M.C. Shih, R.M.H. New, R.F.W. Pease, R.L. White, J. Appl. Phys. 80 (1996) 342. 
[13] R.M.H. New, R.F.W. Pease, R.L. White, J. Vac. Sci. Technol. B 13 (1995) 1089.

[14] A. Fernandez, P.J. Bedrossian, S.L. Baker, S.P. Vernon, D.R. Kania, IEEE Trans. Magn. 32 (1996) 4472.

[15] A. Fernandez, H.T. Nguyen, J.A. Britten, R.D. Boyd, D.R. Kania, A.M. Hawryluk, M.D. Perry, J. Vac. Sci. Technol. B 15 (1997) 729.

[16] A. Fernandez, J.Y. Decker, S.M. Herman, D.W. Phillion, D.W. Sweeney, M.D. Perry, J, Vac. Sci. Technol. B 15 (1997) 2439.

[17] V. Elings, J. Gurley, U.S. Patent No. 5,308,974, Digital Instruments, Santa Barbara, CA.
[18] M.R. Gibbons, J. Magn. Magn. Mater., acceptedfor publication (1998). $186(1998) 38 \%$.

[19] K. Babcock, M. Dugas, S. Manalis, V. Elings, Mater. Res. Soc. Symp. Proc. 355 (1995) 311.

[20] D.R. Fredkin, T.R. Koehler, J. Appl. Phys. 67 (1990) 5544.

[21]_MR Gibons, Fen G. AQ Pl. (1998),-stromitted for publieation.

[22] G.A. Gibson, S. Shultz, J. Appl. Phys. 73 (1993) 4516.

[23] W. Wernsdorfer, K. Hasselbach, A. Sulpice, A. Benoit, J.-E. W w indrawen : Wegrowe, L. Thomas, B. Barbara, D. Mailly, Phys. Rev. B. 53 (1996) 3341.
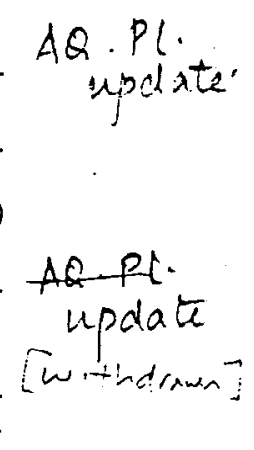